\title{
Les pratiques économiques des entrepreneurs issus de l'immigration chinoise. Le cas de Paris, Bruxelles et Montréal
}

Economic practices of Chinese immigrants entrepreneurs. The case of Paris,

Brussels and Montreal

Las prácticas económicas de los empresarios de la inmigración china. El caso de

París, Bruselas y Montreal

\section{Alexander Nicholls}

\section{(2) OpenEdition}

\section{Journals}

Édition électronique

URL : https://journals.openedition.org/remi/7143

DOI : 10.4000/remi.7143

ISSN : $1777-5418$

Éditeur

Université de Poitiers

Édition imprimée

Date de publication : 1 janvier 2015

Pagination : 149-173

ISBN : 979-10-90426-24-5

ISSN : 0765-0752

\section{Référence électronique}

Alexander Nicholls, «Les pratiques économiques des entrepreneurs issus de l'immigration chinoise. Le cas de Paris, Bruxelles et Montréal », Revue européenne des migrations internationales [En ligne], vol. $31-n^{\circ} 1$ | 2015, mis en ligne le 01 janvier 2018, consulté le 15 avril 2022. URL : http://

journals.openedition.org/remi/7143; DOI : https://doi.org/10.4000/remi.7143 


\title{
Les pratiques économiques des entre- preneurs issus de l'immigration chinoise. Le cas de Paris, Bruxelles et Montréal
}

\author{
Alexander Nicholls ${ }^{1}$
}

\section{Introduction}

La pratique du commerce par les minorités issues de l'immigration peut être propice au développement d'échanges économiques entre entreprises de co-ethniques, du niveau local au niveau transnational. Ce phénomène a été observé dans différents lieux et époques chez les entrepreneurs issus de l'immigration chinoise dite " ethnique ". Dès lors, doit-il être vu comme culturel ou relevant d'une pure rationalité instrumentale ? Doit-on penser cette pratique comme statique et constante, ou fortement corrélée à des contingences historiques, politiques, ou bien géographiques ? Sans prétendre apporter de réponse définitive à ces questions, cet article a pour objectif de les explorer à partir des résultats d'une enquête de terrain effectuée auprès de soixante entreprises liées aux populations chinoises des villes de Paris, Bruxelles et Montréal. Partant de ce matériau empirique, I'article aborde le sujet des petites entreprises des immigrés chinois sous l'angle de la répartition des échanges économiques selon un critère géographique, sachant que les liens économiques étudiés consistent en des relations entre entreprises dans le cadre d'approvisionnement ou de vente de biens et de services. Dans une première partie, les concepts et théories sur l'entrepreneuriat dit " ethnique " ou " immigré " composent le cœur de la réflexion, l'auteur insiste sur la complexité du phénomène et du contexte impliqué. Ce point de départ constitue une trame de fond pour la deuxième partie, au sein de laquelle sont présentés l'enquête et les terrains d'investigation. L'ensemble introduit des données empiriques concernant les liens nationaux et internationaux des entreprises étudiées qui sont comparées, dans une troisième partie, selon les caractéristiques du patron (âge, génération, genre) et de la ville d'implantation. La recherche comporte toutefois des limites importantes, principalement du fait de la grande diversité de l'échantillon. En effet, seul un petit

1 Docteur en sciences politiques et sociales, université catholique de Louvain,

3/4 Place de I'Alma, 1200 Bruxelles ; alexsn2010@gmail.com 
nombre de personnes possède un même dialecte, un même secteur d'activité ou un parcours semblable qui permettrait une comparaison approfondie entre les pays. Le lecteur ne perdra donc pas de vue le fait que les pratiques économiques décrites et analysées le sont à partir d'un nombre réduit d'observations, en aucun cas généralisables à l'ensemble des commerçants chinois.

\section{Cadre théorique et présentation de l'enquête}

\section{Entrepreneuriat immigré : concepts et théories}

Que ce soit en Europe occidentale ou en Amérique du Nord, les études montrent que les populations d'origine immigrée sont fortement représentées dans certains secteurs d'activités commerciales telles que le commerce de proximité, la restauration ou encore l'import-export (Pécoud, 2012 : 2). Light et Karageorgis (1994: 649) différencient trois types de groupes ethniques minoritaires selon qu'ils présentent un taux d'entrepreneuriat supérieur, comparable ou inférieur à la moyenne nationale; les Chinois, dont il est question dans cet article, font partie du premier et ceci s'est constaté dans plusieurs pays d'immigration, précisent. Plusieurs théories ont été confrontées pour expliquer ce phénomène : traits culturels spécifiques (Berger, 1991 ; Redding, 1991), mobilité bloquée ( $\mathrm{Li}, 1993$; Auster et Aldrich, 1984), position de non-membre dans la société des minorités intermédiaires (Bonacich, 1973), économie d'enclave (Salaff et al., 2003 ; Dyer et Ross, 2000 ; Wilson et Portes, 1980). Elles ont pu être centrées sur le contexte (social et géographique), la classe sociale (capital financier et compétences) ou l'ethnicité (ressources ethniques) de l'entrepreneur (Pécoud, $2012: 2$ ).

D'autres travaux, qui sont ici privilégiés, se veulent plus complexes et nuancés. Citons la théorie de l'encastrement de la sphère économique dans le social de Granovetter $(1985 ; 2006)$ et le modèle multifactoriel et interactif de Waldinger, Aldrich et Ward (1990) selon lequel le développement du commerce dépend d'interactions complexes entre les stratégies de l'acteur, les structures économiques et les ressources du groupe. Ce modèle combine les approches contextuelle et ethnique (Pécoud, 2012 : 4). Dans la continuité de l'effort de théorisation fourni par Waldinger et al. (1990), une théorie de l'encastrement mixte a pu être proposée par Kloosterman et Rath $(2003 ; 2001)$ dans la perspective de comprendre l'entrepreneuriat des immigrés en localisant le phénomène dans son milieu socioéconomique. Les marchés s'insèrent, en effet, dans des contextes sociaux plus larges et diffèrent selon les lieux et les époques; dès lors, explorer l'entrepreneuriat immigré dans différents pays nécessite une comparaison des structures d'opportunités. Souhaitant faciliter les études comparatives entre les pays, Kloosterman et Rath (2001 : 193-198) proposent une approche à trois niveaux : national (lois, institutions, marchés, culture), régional ou urbain (économie de la ville, programmes urbains) et local ou du voisinage (taux de population immigrée, présence de commerces). Dans la lignée de Waldinger, ils lient les concepts de structure et d'agentivité (Kloosterman et Rath, $2003: 8$ ).

Toutefois, contrairement à Waldinger, ces auteurs ne parlent plus d'entrepreneuriat " ethnique ", mais " immigré ", tout en reconnaissant la difficulté 
à définir ce concept, tributaire de la définition nationale (/bid., $2003: 4-5)$. Par ailleurs, la complexité observée en matière de commerce dit "ethnique " amène à considérer cette notion avec prudence. Souvent employée " de manière extensive sans une observation fine des échanges économiques " (Berbagui, 2005 : 113), son champ d'application varie fortement selon les auteurs. Pour certains, tout commerce tenu par un membre d'une minorité ethnique peut être qualifié de tel (Auster et Aldrich, $1984: 49$ ) alors que d'autres réservent ce terme aux commerces associés à une communauté ethnique dont les marchés seraient limités et spécialisés ( $\mathrm{Li}, 1988: 52)$ et que d'autres encore l'appliquent à tout commerce dont les patrons et employés appartiennent à une même communauté ethnique (Light et Karageorgis, 1994 : 647-649). Pécoud (2012) classe les définitions en trois types : selon le premier, le commerce est dit ethnique lorsque le patron appartient au groupe ethnique parce qu'il s'y identifie ou qu'il y est identifié, selon le deuxième, le caractère relationnel de l'ethnicité est déterminant, l'enjeu n'est pas l'appartenance d'un individu, mais les relations entre personnes (employeur et employés, commerçant et clients) partageant cette appartenance, tandis que le troisième type se concentre sur l'impact de l'ethnicité sur le commerce et la manière dont les économies ethniques sont façonnées par les caractéristiques socioculturelles du groupe concerné. Le concept de commerce ethnique est donc controversé, si bien que des auteurs comme Zalc (2010) en interrogent la pertinence. Selon cette chercheuse, l'activité commerçante est la cause plutôt que la conséquence de solidarités dites " ethniques ", lesquelles sont liées à un contexte particulier d'immigration et d'expériences professionnelles similaires plutôt qu'à des origines communes. Pécoud, quant à lui, souligne l'aspect non neutre de ce terme, en effet, " qualifier

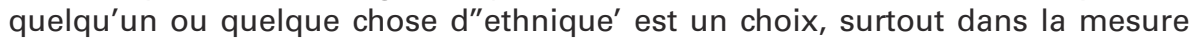
où tous les immigrés ne sont pas appelés 'ethniques' "(Pécoud, 2012 : 7). Sans nier le rôle des liens communautaires, il convient d'éviter d'assimiler l'entrepreneuriat immigré à l'utilisation de réseaux ethniques, laquelle est loin d'être systématique (/bid. : 14-17).

Il est également important de souligner que l'ethnicité et l'économie immigrée sont traversées par des clivages internes comme le genre, la génération ou encore la classe sociale (Pécoud, 2012 : 14). Si la dimension familiale de ces petites entreprises a souvent été étudiée (Smart, 2003 ; Zalc, 2001 ; Light et Karageorgis, 1994 ; Berger, 1991 ; Redding, 1991), les théories culturalistes sur l'entrepreneuriat occultant les rapports de pouvoir au sein de ces populations, notamment l'exploitation des femmes, des jeunes générations et des immigrés clandestins (Nonini et Ong, 1997) sont à considérer avec précaution. Selon Phizacklea, la variable " genre " est souvent oubliée dans les études sur le sujet. Pour les hommes immigrés, l'entrepreneuriat est un moyen d'échapper aux emplois pénibles dans lesquels ils sont confinés, alors que les femmes restent cantonnées dans les emplois subalternes. L'entreprise demeure patriarcale dans le sens matérialiste du terme : elle est un lieu d'appropriation du travail des femmes par les hommes (Phizacklea, 1988 : 20-22). Dans les commerces familiaux, les décisions sont prises par les hommes âgés tandis que les hommes jeunes et les femmes effectuent la plupart du travail (Greenhalgh, 1994 ; Phizacklea, 1988). Phizacklea en conclut qu'en fonction du genre et de la génération, les membres d'une même famille ne partagent pas la même classe sociale (Phizacklea, $1988: 24)$. D'autres études se sont éloignées de la tendance 
à décrire les femmes comme passives et dépendantes en s'attachant à mettre en évidence leurs stratégies en tant qu'entrepreneures (Morokvasic, 1991) ou employées dans la firme familiale (Smart, 2003).

Enfin, les frontières externes du groupe considéré évoluent en fonction des interactions avec la majorité et d'autres groupes immigrés, et ceci dans un contexte institutionnel, social et politique variant selon le pays. L'ensemble incite donc à un usage raisonné, prudent et critique des labels ethniques (Pécoud, $2012: 14-15)$.

\section{Une enquête auprès de "commerces chinois "}

Précisons d'abord ce que recouvrent, dans le cadre de cette recherche, les termes de " commerce chinois " et "commerçant chinois ", expressions parfois utilisées dans une optique culturaliste, voire essentialisante. Bien que reconnaissant l'importance des éléments culturels, notre approche de l'ethnicité se veut constructiviste et interactionniste. La perspective retenue est celle de Barth ([1969] 1995) et Juteau $(1996 ; 1983)$ : le terme " Chinois " est ici utilisé dans son acceptation ethnique, soit comme une construction autour de frontières internes et externes. C'est la raison pour laquelle, dans la sélection des personnes interrogées (soixante en tout2), nous avons pris le parti de nous en référer à leur autodéfinition en tant que "Chinois ". Originaires de la République populaire de Chine, de Taïwan ou d'Asie du Sud-Est, ces personnes se reconnaissent des ancêtres communs. Gardons toutefois à l'esprit le phénomène de " saillance " ou la mise en valeur contextuelle d'un élément identitaire (Poutignat et StreiffFenart, 1995), ici une relation d'enquête avec un chercheur " non chinois ", membre de ce que Wirth (1945) appelait le " groupe majoritaire ". II est toutefois remarquable que plusieurs répondants aient d'eux-mêmes insisté sur l'importance pour les Chinois de conserver une certaine cohésion en situation d'immigration, notamment à travers les associations, les écoles de langue chinoise, ou encore le maintien de traditions culturelles et religieuses (Nicholls, 2011, 2012).

Un sentiment d'appartenance ethnique est donc ce qui lie ces soixante personnes, arrivées entre les années 1950 et 2000 dans les villes de Paris, Bruxelles et Montréal. villes. Ces enquêtés se situent de fait, à des moments différents de leur parcours migratoire et, par conséquent, de leurs activités professionnelles. Concernant le sous-groupe que composent les entrepreneurs, toutes les expériences sont considérées et il peut donc s'agir de personnes interrogées au cours de leur première année d'installation ou à l'inverse au terme d'une longue période d'activité. Si les entretiens réalisés auprès de patrons, de conjoints ou d'enfants adultes travaillant dans le commerce familial ont été privilégiés, un peu plus d'un quart des entretiens a été effectué auprès d'un employé de confiance, extra-familial, référé par le patron. Les entreprises sont, elles aussi, variées : restaurants, commerces alimentaires, herboristeries, boutiques de décoration, magasins de vêtements, d'accessoires de mode, agences de

\footnotetext{
2 Les enquêtés se distribuent ainsi à Paris (17), Bruxelles (18) et Montréal (25). II s'agit d'hommes (60\%) et de femmes (40\%), de 18 à 84 ans (de 30 à 84 ans, en ne comptant que les patrons ou anciens patrons), en provenance de divers pays (Chine, Taïwan, Vietnam, Cambodge, Laos, Thaïlande, Indonésie, Madagascar) et s'exprimant en différents dialectes (mandarin, cantonais, chaozhou, wenzhou, qingtian, shanghaïen, fukien, changsha).
} 
voyage, salons de coiffure, bijouteries, horlogeries et autres. La plupart d'entre elles entrent dans la catégorie des très petites entreprises, compte tenu de leur chiffre d'affaire (moins de deux millions d'euros) et du nombre d'employés (moins de vingt). Seules deux entreprises montréalaises appartiennent à celle des petites et moyennes entreprises (c'est-à-dire ayant un chiffre d'affaires de moins de 43 millions d'euros et moins de 250 employés) : un grand restaurant et une pâtisserie industrielle. Deux voies ont été privilégiées afin de réunir ces entretiens : la première consistant en l'exploitation de réseaux sociaux, à partir d'associations ou de personnes-ressources, la seconde, en faisant du porte à porte dans les zones de regroupement de commerces tenus par des Chinois. Afin d'allier récolte de données objectives sur l'existence et la nature des partenaires économiques et approche compréhensive axée sur la subjectivité des acteurs (Kaufmann, 1996), nous avons opté pour l'entretien semi-directif. Celui-ci consiste en des questions ouvertes s'orientant autour de grands axes: le parcours migratoire, le parcours professionnel de la création du commerce à ses perspectives de développement, l'insertion du commerce dans l'économie ethnique locale et transnationale, la clientèle, les rapports intracommunautaires et interethniques avec la majorité. La méthode d'analyse est qualitative, bien qu'incluant quelques statistiques de base sur les personnes interrogées.

\section{Paris, Bruxelles et Montréal comme terrains d'enquête}

Partant de l'idée que l'État, acteur ethnique légitimant la culture des majoritaires et gérant la diversité du pays (Bastenier, 2004 : 193), est susceptible d'influencer le comportement des minorités, l'approche comparative est pertinente pour conduire l'analyse des pratiques économiques de ces acteurs sociaux au sein de trois métropoles occidentales, à dominance francophone et, dans les faits, multiculturelles, mais comprenant toutefois quelques différences. Ces dernières sont d'ordre philosophique et politique, en premier lieu. Ainsi, l'égalité des cultures, base de la politique fédérale canadienne du multiculturalisme et l'échange entre les cultures, base de la politique québécoise de l'interculturalisme (Cardinal et Couture, 1998 ; Juteau et al., 1998 ; Kymlicka, 1995 ; Taylor, 1994) sont rejetés en France au nom du principe républicain qui réserve à la sphère du privé les questions liées à la diversité culturelle et religieuse (Noiriel, 2001 ; Schnapper, 1994), tandis que la Belgique se distingue par une approche pragmatique reconnaissant des " communautés culturelles " dans le cadre d'infra-politiques inspirées du multiculturalisme (Martiniello, 1993 ; Bastenier, 1992). Les politiques migratoires diffèrent également : le Canada prévoit la possibilité d'immigrer sur base de ses études, de son expérience professionnelle ou en tant qu'investisseur ( $\mathrm{Li}, 1988$ ), alors que la France et la Belgique ont, depuis 1974, mis en place une politique de contrôle strict des flux migratoires (Hullebroek, 1992 ; Milza, 1985). Ces disparités sont également d'ordre démographique. Au Canada, la population se déclarant d'ethnicité chinoise compte plus de 1200000 personnes au recensement de 2006, soit 3,5\% de la population totale, dont 72000 enregistrées à l'échelle du " grand Montréal " (Statistique Canada, 2006). La population chinoise ethnique en France est, quant à elle estimée à 300000 personnes par l'ambassade de Chine (Yun et Poisson, 2005). Ce groupe compose $0,5 \%$ de la population du pays, et la plupart de ses membres résident à Paris, principalement dans les XIII (réfugiés d'Asie du Sud-Est), III (filière du Zhejiang), $\mathrm{XI}^{\mathrm{e}}$ et $\mathrm{XII}^{\mathrm{e}}$ arrondissements et dans le secteur de Belleville (Ma Mung, 2000). En Belgique, cette population est estimée à 
23000 personnes, soit $0,2 \%$ de la population globale, dont un peu moins du tiers est installé à Bruxelles (CECLR, 2005 ; Pang, 2002). Précisons que la France et la Belgique ne connaissent pas de statistiques ethniques et que ces chiffres sont bien supérieurs aux statistiques officielles ne recensant que les personnes de nationalité chinoise, dont le nombre est de plus de 72000 pour la première (INSEE, 2007) et d'environ 7500 pour la seconde (Institut national de la statistique, 2006).

Quel que soit le pays, une augmentation du nombre d'immigrés, en provenance de pays variés, est notable. Pour Kloosterman et Rath, cela se traduit par davantage de candidats immigrés-entrepreneurs, aux pratiques de plus en plus internationales. Dans les économies post-industrielles ou post-fordistes des pays développés, les manufactures déclinent et le secteur tertiaire prend de I'importance. Le secteur des services aux personnes et aux entreprises s'affirme (Kloosterman et Rath, 2003 : 10-12) et représente de nouvelles opportunités pour les commerces immigrés (Kloosterman et Rath, 2001 : 193). Cependant, la création d'entreprises dans ce contexte n'est pas toujours un choix; elle résulte également de la crise de l'État-providence et de l'emploi, des restructurations industrielles et de l'augmentation du taux de chômage (Pécoud, $2012: 2$ ). Si Kloosterman et Rath (2003 : 1-2) reconnaissent l'existence de barrières à I'encontre des immigrés et le rôle des ressources collectives ou ethniques, ce microcontexte ne saurait éclipser l'existence de macrofacteurs politiques, légaux, économiques et institutionnels dont les immigrés-entrepreneurs dépendent de manière beaucoup plus passive. Le contexte politico-légal, responsable des lois qui encadrent la création et le fonctionnement des commerces, ainsi que les qualifications requises pour devenir commerçant varient d'un pays à l'autre. Le contexte économico-institutionnel, quant à lui, dépend de la manière dont les États et les acteurs sociaux (patronat, syndicats, chambres de commerce et de l'industrie, notamment) régulent institutionnellement les activités économiques. II en résulte que l'environnement nord-américain est idéologiquement et contextuellement plus favorable à la création d'entreprises que les pays d'Europe continentale qui contrôlent fortement les activités commerciales (Pécoud, 2004 : 20-23). Ce dernier point a été confirmé par le discours des entrepreneurs chinois de Montréal, bien plus nombreux à expliquer la création d'entreprises par leur souhait d'être leur propre patron, que résultant d'une difficulté à s'insérer dans le marché de l'emploi, un argument davantage mis en avant à Paris et Bruxelles (Nicholls, 2011).

À l'échelle infra-urbaine des trois métropoles, des différences sont à noter. II existe à Montréal une reconnaissance officielle d'un "Chinatown " au centre-ville, même si majorité des commerces se trouve à l'extérieur de cet espace ${ }^{3}$. Pour Cha (2004), la construction des arches délimitant le quartier et l'habillage de celui-ci est un acte d'appropriation spatiale sans précédent à Montréal. À Paris, plusieurs zones de regroupement de commerces chinois et asiatiques sont recensées, les plus importantes se situent dans le XIII et le III arrondissements. À Bruxelles, enfin, les commerces chinois semblent se limiter aux traditionnels restaurants de quartier, bien qu' un îlot asiatique soit identifié au centre-ville (Nicholls, 2011).

3 Un Chinatown reconnu comme patrimoine culturel par les autorités de la ville s'étend aux alentours de la rue La Gauchetière (Cha, 2004). 


\section{Les échanges économiques ethniques au niveau national et transnational}

Les liens ou réseaux économiques ethniques dont il est ici question sont identifiés par la présence chez les entreprises étudiées de partenaires commerciaux co-ethniques parmi leurs fournisseurs ou leurs entreprises clientes. Nous avons classé ces derniers selon la localisation de leur établissement dans la même ville ou, plus rarement, le même pays (niveau national), voire un pays étranger (niveau transnational).

\section{Résultats de l'enquête}

\section{Au niveau national}

Sur les soixante entreprises de notre échantillon, un peu plus de la moitié (trente-deux) entretient des liens économiques avec d'autres commerces de co-ethniques installés dans le pays d'accueil (tableau 1). C'est ce que nous nommons les " réseaux nationaux". Notons que le " oui " couvre une variété de situations pour l'entrepreneur : la collaboration avec des co-ethniques pouvant être de faible à quasi-exclusive.

Tableau 1 : Présence de réseaux économiques nationaux

\begin{tabular}{|l|l|l|l|l|}
\hline & Bruxelles & Paris & Montréal & Total \\
\hline Oui & 9 & 9 & 14 & 32 \\
\hline Non & 8 & 9 & 11 & 28 \\
\hline Total & 17 & 18 & 25 & 60 \\
\hline
\end{tabular}

Cette vue d'ensemble indique que nos répondants montréalais ont davantage de collaborateurs co-ethniques dans le même pays. Cette légère différence de résultats s'explique, à travers le contenu des entretiens, par le grand nombre d'entrepreneurs chinois installés dans les villes de Toronto et Vancouver et l'import-export de produits entre villes canadiennes. Les entreprises concernées par ce type d'importation ne sont qu'au nombre de trois dans notre échantillon. II $\mathrm{s}^{\prime}$ agit d'un revendeur d'objets décoratifs $\left(\mathrm{M}\right.$. Qian ${ }^{4}$ ) se fournissant partiellement en Chine et dans ces deux villes, d'un herboriste (M. Bian) se fournissant pour $70 \%$ en Chine, pour $30 \%$ à Vancouver et d'un magasin de vêtements s'approvisionnant pour moitié à Toronto :

"Pour [l'approvisionnement], ma femme connait des entreprises chinoises... II y a des choses qui viennent de Chine et d'autres qui viennent de Toronto... Pour le moment, c'est à peu près moitié-moitié. [...] Parce qu'à Toronto, il y a aussi beaucoup de Chinois et on visite beaucoup là-bas ! "(M. Wang, époux et employé de Mme Wang)

Un quatrième cas est celui d'une pâtisserie industrielle tenue par un Chinois dont nous avons interrogé un employé co-ethnique. Celle-ci approvisionne des petits commerces alimentaires de Toronto et Vancouver ; seul cas rencontré d'exportation à partir de Montréal.

4 Tous les noms des personnes interrogées mentionnées dans cet article ont été modifiés. 
Les liens entre les entreprises montréalaises, quant à eux, ne sont guère plus nombreux que ceux décomptés à Paris et Bruxelles. Parmi les personnes interrogées, nous trouvons des grossistes et détaillants, des restaurateurs et autres commerçants d'alimentation " exotique " ainsi que diverses entreprises (éditeur de journaux chinois, libraires). Les discours des répondants de Paris indiquent que les liens entre les entreprises locales de co-ethniques y sont proportionnellement moins développés par rapport aux entreprises locales tenues par des Français ou d'autres immigrés, un constat étonnant au regard de l'importance numérique de ces communautés chinoises. Dans le XIIle arrondissement, les liens entre co-ethniques apparus lors de l'enquête impliquent des restaurants s'approvisionnant auprès de grandes surfaces, des détaillants auprès de grossistes et vice-versa, ou encore un journal chinois achalandant des libraires. Les grossistes wenzhous du III arrondissement n'accordent que peu d'importance à I'origine ethnique de leurs détaillants, ainsi vendre à des co-ethniques est, chez eux, une pratique minoritaire, voire inexistante.

"Mes clients, ce sont des Européens avec des magasins de produits occidentaux. I/s cherchent juste des accessoires pour pas cher. [...] Ce sont [des] Européens à 100\%!"

(M. Cao, grossiste en bijoux fantaisie et accessoires de mode)

Une répondante nous fait part de sa préférence pour les détaillants français:

"[C]e sont des Français, des Chinois, des Africains... II y a de tout ! [...] Avec les Français, c'est plus facile ! Les Asiatiques, eux, ils demandent de baisser les prix [...].

Les Français, ils ne demandent pas ça, ils sont très gentils. Et quand il y a une fête ou quoi, moi je donne un cadeau et eux ils disent: 'Non, non, non !'. Ils sont très gentils !

Les Africains, c'est le contraire : si tu fais un cadeau, ils demandent plus, plus, plus ! Eux aussi, c'est difficile ! Les Français se sont les plus faciles comme clients. " (Mme Zheng, grossiste en maroquinerie et objets touristiques)

Alors que les grossistes du $\mathrm{III}^{\mathrm{e}}$ arrondissement sont dans un schéma de cohabitation sans interconnexion, le quartier du $\mathrm{XIII}^{\mathrm{e}}$ présente davantage de relations entre les commerces, principalement entre grossistes et détaillants, et entre restaurants et commerces alimentaires. À Bruxelles où le paysage des commerces chinois est dominé par la restauration, c'est sans surprise que nous découvrons que les restaurateurs interrogés sont dépendants de supermarchés importants de denrées chinoises et asiatiques sur le sol belge, tandis que les commerces non alimentaires restent isolés par rapport aux autres entreprises chinoises de la ville. La taille restreinte de la communauté chinoise a même incité les restaurateurs, dans un passé récent, à établir des contacts avec des commerces chinois d'autres villes. Ainsi, Mme Tran, sino-vietnamienne et fille de restaurateurs, nous explique qu'au début des années 1980, ses parents se procuraient la décoration auprès de Chinois des Pays-Bas et la nourriture auprès de Chinois d'Anvers, ceci avant l'ouverture d'épiceries asiatiques à Bruxelles. Le cas des restaurants chinois et asiatiques mérite d'ailleurs un développement particulier. La provenance des produits est toujours mixte. Les denrées sont achetées à des Chinois et à des Occidentaux selon les prix et le type de produits. Les aliments périssables (viande, légumes) sont achetés sur place, souvent à des Occidentaux (par exemple, en allant au marché le matin), alors que les aliments typiquement chinois ou asiatiques sont achetés à des grandes surfaces et à des grossistes. 
"[I]l y a deux façons. On commande chez le grossiste ; là, il y a, par exemple, le riz, la farine, et certains aliments secs. Là, vous achetez chez le fournisseur ; il y a les fournisseurs belges et les fournisseurs chinois, aussi. Et puis tous les produits qu'on peut trouver dans les magasins chinois, mais ce sont des produits [comme] les champignons chinois, les légumes frais ; là, il faut acheter tous les jours. " (M. Huang, restaurateur, Bruxelles)

Interrogés sur leurs préférences, des motivations de type rationnel sont souvent mises en avant :

"Ça dépend de la qualité, ça dépend du prix, ça dépend... C'est pas spéciale-

ment [chez] les Chinois. Mais il y a quelques produits, ce n'est que les Chinois qui [les] vendent : ça, il n'y a pas le choix. Mais si c'est [pour] le vin [...] c'est équivalent, quoi ! Ce n'est pas seulement la nationalité... "(M. Guo, restaurateur, Bruxelles).

"C'est pas seulement chez des Chinois. Par exemple, la viande, je vais plutôt à l'abattoir ; certaines viandes, les gibiers, heu, chez le grossiste en gibier ; les légumes [...] chez le marchand de légumes [...]. Pour certaines choses, évidemment, je suis obligé de m'approvisionner chez les Chinois et d'autres chez les Thaïlandais aussi. [Mais] par exemple, le vin français, je me vois très mal m'approvisionner chez un Chinois...

Et le brasseur, par exemple... " (M. Chow, restaurateur, Bruxelles)

Pour ce dernier point, les restaurateurs du XIII a arrondissement de Paris sont moins réticents à s'approvisionner auprès de commerces alimentaires asiatiques comme les supermarchés Tang Frères:

"On travaille avec le patron qui fait aussi de l'import-export. [...] On travaille aussi avec Tang. Ce sont [nos] fournisseurs [en] vin et boissons. [...] Pour la cuisine, pour commander des viandes autres, des gigots, on commande à des entreprises françaises. "

(M. Chung, employé de restaurant, Paris)

La situation des restaurants dans les trois villes est comparable avec toutefois quelques nuances. Les restaurateurs, tributaires des commerces exotiques pour certaines denrées, sont la plupart du temps inclus de fait dans un réseau économique ethnique. Cependant, la proportion de denrées achetées auprès de co-ethniques est très variable d'un restaurant à l'autre. Pour beaucoup, tels ces deux restaurateurs bruxellois, les produits comme la viande, les légumes ou les boissons proviennent de fournisseurs occidentaux et ils limitent les achats dans les supermarchés et épiceries asiatiques. M. Chung quant à lui indique s'approvisionner de manière plus importante chez des co-ethniques. Cette différence met en évidence l'importance du nombre et de la variété des commerces chinois dans l'établissement de liens économiques.

\section{Au niveau transnational}

En ce qui concerne les échanges internationaux, une petite majorité de trente et un sur soixante importe directement de l'étranger (ta- bleau 2), principalement de Chine et de divers pays d'Asie (tableau 3). 
Tableau 2 : Présence de liens économiques internationaux

\begin{tabular}{|l|l|l|l|l|}
\hline & Bruxelles & Paris & Montréal & Total \\
\hline Oui & 9 & 11 & 11 & 31 \\
\hline Non & 8 & 7 & 13 & 28 \\
\hline Nsp & 0 & 0 & 1 & 1 \\
\hline Total & 17 & 18 & 25 & 60 \\
\hline
\end{tabular}

Tableau 3 : Les pays d'importation selon la ville

\begin{tabular}{|l|l|l|l|l|}
\hline & Bruxelles & Paris & Montréal & Total \\
\hline Co-ethnique Asie & & & & \\
\hline Chine et Hong-Kong & 9 & 10 & 11 & 30 \\
\hline Taïwan & 1 & 0 & 1 & 2 \\
\hline Vietnam & 1 & 0 & 1 & 2 \\
\hline Thaïlande & 1 & 2 & 1 & 4 \\
\hline Indonésie & 0 & 1 & 0 & 1 \\
\hline Asie non spécifié & 0 & 1 & 1 & 2 \\
\hline Non Co-ethnique & & & & \\
\hline Japon & 2 & 2 & 3 & 7 \\
\hline Corée & 1 & 1 & 0 & 2 \\
\hline Co-ethnique Europe & & & & \\
\hline Belgique & N/A & 1 & 0 & 1 \\
\hline Allemagne & 0 & 1 & 0 & 1 \\
\hline Pays-Bas & 1 & 0 & 0 & 1 \\
\hline Co-ethnique Amérique & & & & 1 \\
\hline États-Unis & 0 & 0 & 1 & \\
\hline
\end{tabular}

Nous constatons en premier lieu une similitude entre les commerçants des trois villes, au regard de leurs pratiques d'importation. La Chine est le pays d'importation d'une écrasante majorité d'entre eux (30 sur 31), laissant loin derrière elle les autres pays. Le Japon est cité sept fois, notamment pour l'importation de thé et d'objets décoratifs, témoignant de l'importance pour certains types de commerces des réseaux transnationaux non ethniques. D'autres pays dont nos répondants sont originaires (Taïwan, Vietnam, Indonésie, Thaïlande) ne sont cités que de manière marginale, ceci malgré la présence importante de réfugiés d'Asie du Sud-Est. Ces derniers font essentiellement commerce avec la Chine, une tendance qui s'accentue au fil du temps.

" J'ai ouvert en 1992. [...] Puis, pendant 10 ans, j'ai fait venir de Philippines, de Thaïlande, de Hong Kong, de différents pays. [...] Maintenant, c'est 100\% en Chine.

Parce que ça va pas dans les autres pays : c'est très cher. " (Mme Xu, magasin de bibelots et vêtements, Montréal)

Cette tendance ne s'est cependant pas généralisée. Ainsi, à Paris, si M. Tang et Mme Zheng importent l'entièreté de la marchandise de Chine, 
d'autres comme M. Cao se fournissent principalement en Asie du Sud-Est. Notre enquête a aussi permis de mettre en évidence le cas de M. Yao, ébéniste et vendeur de meubles, qui s'approvisionne auprès des entreprises créées par sa mère en Indonésie et en Chine, à la fin des années 1980 :

"Nous avons une entreprise en Chine, [une] en Indonésie, et puis tous les articles viennent de là-bas, surtout tout ce qui est en bois, tout ce qui est meubles, bois et petites décorations. [...] Nous avons une entreprise qui fabrique les meubles en Indonésie, en teck et en acajou, et puis nous exportons ça la plupart du temps en Europe, surtout en Allemagne, et puis nous avons également une entreprise en Chine qui fabrique les meubles asiatiques, tout ça, des copies, ou voire des récupérations de pièces antiques [pour] les restaurer et réexpédier ça en Allemagne, en Angleterre ou même aux ÉtatsUnis. Et puis, je vois les perspectives en France au niveau de cette tendance de meubles qui plaît à beaucoup de monde. [...] C'est pourquoi j'ai [créé] une boutique de meubles de décoration. [...] Actuellement, je fais [venir] 70\% de Chine, 20\% de I'Indonésie et 10\% dans le reste de l'Asie du Sud-est. [...] Je n'achète jamais aux entreprises chinoises en France parce que tous les articles sont disponibles dans les entreprises de Chine et d'Indonésie. "

L'importance croissante de l'économie transnationale, sur laquelle nous reviendrons ultérieurement, apparaît également lorsque nous croisons les données sur les fournisseurs nationaux et transnationaux de ces entreprises. Le cas le plus fréquent (dix-sept entreprises) est, en effet, l'approvisionnement auprès d'entreprises de co-ethniques situées à l'étranger combiné à l'absence ou à la marginalité (moins de $10 \%$ des marchandises) du ravitaillement national. Montréal se distingue, toutefois, en raison des entreprises de confection de Vancouver et Toronto. La part d'approvisionnement issue de fournisseurs nationaux et transnationaux se révèle plus équilibrée, beaucoup de répondants faisant part d'un apport conséquent des fournisseurs internes au pays.

\section{Les réseaux économiques selon la ville}

Si des différences entre nos terrains d'enquête sont observables, parler de différences entre les villes est insuffisant puisqu'une même ville rassemble toujours plusieurs communautés chinoises, différant par leur provenance et leur histoire migratoire. Ceci est particulièrement visible à Paris qui connaît un clivage affirmé entre la population chinoise du XIII arrondissement, majoritairement chaozhou et issue de l'Asie du Sud-Est et la population wenzhou du $\mathrm{III}^{\mathrm{e}}$ arrondissement. Tandis que les premiers ont privilégié des relations entre leurs commerces, notamment entre grossistes et détaillants et entre supermarchés et restaurateurs, les seconds ont misé sur d'importants réseaux ethniques transnationaux. En outre, comme nous l'avons vu à travers les témoignages, les grossistes wenzhous fournissent essentiellement des détaillants non chinois.

À Bruxelles, les liens économiques locaux sont relativement récents. Ainsi, avant l'ouverture du Sun Wah de Bruxelles en 1985 (devenu le Kam Yuen en 2002), les aliments asiatiques étaient importés de Paris ou d'Anvers par les restaurateurs. Les patrons d'autres types de commerce (boutiques de vêtements, de bijoux, de remèdes chinois, d'objets décoratifs et de jouets) s'approvisionnent uniquement via des réseaux transnationaux; ceux-ci sont principalement co-ethniques et en provenance de Chine continentale, plus rarement japonais 
ou coréens. Seule une créatrice et vendeuse de bijoux artisanaux déclare ne pas avoir de réseaux économiques ethniques et traiter uniquement avec des "Belges ou Européens"(Mme Lin). À Montréal, enfin, nous avons rencontré plus de variétés dans les pratiques économiques. Comme nous I'avons déjà signalé, les communautés chinoises des villes de Toronto et Vancouver ont développé des entreprises de confection (notamment de denrées alimentaires, de plantes médicinales, d'objets décoratifs, de vêtements) qui fournissent nombre de commerces chinois dans tout le pays, y compris ceux de Montréal. A contrario, de petites entreprises sans caractère exotique (un studio de photographie, une épicerie de type occidental, un fleuriste) ne possèdent aucun lien avec d'autres entreprises chinoises. En effet, si nos répondants montréalais sont fort présents dans la catégorie qui établit des contacts à la fois locaux et transnationaux, ils sont aussi les plus nombreux à ne pas avoir de lien économique ni local ni transnational avec d'autres entrepreneurs chinois. Les différences observées entre les villes dévoilent l'importance de facteurs contextuels comme le poids démographique d'une communauté, l'histoire de son implantation, la variété de ses commerces et la localisation de ceux-ci dans le développement de réseaux économiques ethniques. L'impact du facteur démographique se fait surtout ressentir à Bruxelles avec l'isolement de nombreux commerces chinois. Enfin, les pratiques économiques observées dans ces trois villes dessinent une même tendance qui voit un développement des réseaux transnationaux et la part grandissante de la Chine dans ceux-ci.

\section{Le commerce, une pratique en constante évolution}

L'analyse des pratiques commerçantes issues de nos entretiens souligne l'affirmation sur ces dernières années d'une évolution des pratiques, qui voit être recomposés les rapports économiques entretenus entre les échelles nationale et transnationale. Une évolution liée à la capacité des commerces à se maintenir dans le contexte d'une société en changement, de la ville et de la société d'accueil, mais aussi de la Chine et des pays d'Asie. L'entreprise se situe, dans un contexte socio-économique mondial (Ma Mung, 2009) en perpétuelle évolution et cela se traduit par des adaptations et aménagements des pratiques commerçantes.

\section{Comparaison des pratiques selon les caractéristiques du patron}

L'analyse de discours de nos répondants nous a permis de constater quelques différences selon certaines caractéristiques du patron, tels l'âge, la génération et le genre.

Les entrepreneurs âgés de première génération entretiennent davantage de liens économiques avec les entreprises chinoises locales. De fait, vingt-quatre des vingt-sept patrons présentant ce type de réseaux sont des immigrés de première génération dont la majorité a plus de cinquante ans. Les plus jeunes ont, quant à eux, tendance à délaisser les réseaux locaux au profit des réseaux transnationaux. Ainsi, la quasi-totalité des patrons de moins de quarante ans (12 sur14) se passe de collaboration avec des entreprises de co-ethniques aux niveaux local et national, alors que huit d'entre eux ont établi des liens avec des entreprises chinoises à l'échelle internationale uniquement. Dans l'ensemble, 
les patrons de deuxième génération ont développé de nouvelles pratiques, y compris dans l'établissement de liens économiques. Ces derniers tendent à s'éloigner des réseaux entre patrons chinois d'une même ville, en augmentant les liens transnationaux, majoritairement ethniques, ou encore locaux nonethniques. De même, si certains jeunes patrons entretiennent des réseaux économiques avec d'autres collègues chinois du pays, l'usage qu'ils en font diffère de celui de leurs aînés, comme nous l'avons vu avec l'exemple de Mme Wang (Montréal), s'approvisionnant auprès d'entreprises chinoises de Toronto. Les patrons des jeunes générations ont globalement une meilleure connaissance du pays d'accueil, de ses lois et procédures, des langues et des mentalités. Étant souvent plus éduqués, leur appréhension de l'économie mondiale, leur permet de développer des réseaux transnationaux et leur offre plus de choix dans les pays d'importation. Les transformations opérées par ces jeunes patrons sont aussi d'ordre technique et technologique, avec l'utilisation de l'informatique et de nouveaux moyens de communication (Nicholls, 2011).

Un autre point de divergence observable concerne la différence entre hommes et femmes dans la mobilisation de ressources financières à l'ouverture du commerce ainsi que la rareté de l'aide apportée par le conjoint aux femmes (Nicholls, 2012). Nos résultats confirment le point de vue de Morokvasic (1991) lorsqu'elle affirme que les femmes font face à des obstacles spécifiques, en particulier dans la mobilisation moindre des ressources propres à leur communauté, les incitant à se tourner vers des ressources extra-ethniques. En ce qui concerne les liens économiques avec des entreprises de co-ethniques, notre enquête n'a pas révélé de différence significative selon le genre du patron, si ce n'est que les femmes sont plus présentes dans la catégorie des moins de quarante ans. Relevons toutefois que certaines d'entre elles ont innové en tant que patronnes, indépendamment de leur âge ou de leur génération. Ainsi, Mme Yang, la doyenne de nos répondants a, dans les années cinquante, œuvré à élever les standards de propreté et de décoration dans les restaurants chinois et introduit des produits encore méconnus à l'époque, tels les germes de soja qu'elle importait. Active dans le milieu associatif, elle a rapidement fait figure de leader dans la communauté chinoise de Bruxelles. Mme Feng, proche de la retraite, a également innové en ouvrant un restaurant de cuisine chinoise authentique (soit non adaptée aux goûts des Occidentaux), chose qui n'existait pas dans la capitale belge à l'époque. Contrairement à leurs aînées, les jeunes femmes se tournent plus souvent vers de nouveaux secteurs ou des secteurs non-ethniques, en partie dû au fait qu'elles sont moins nombreuses à reprendre le commerce parental. C'est le cas de MmeTran, sino-vietnamienne de Bruxelles, qui a vu le restaurant parental repris par son frère et qui a développé une activité de traductrice et de guide touristique indépendante orientée vers une clientèle chinoise.

\section{Comparaison des pratiques selon la ville}

L'aspect géographique, c'est-à-dire la ville et le quartier d'activités, s'est révélé être un facteur déterminant de différenciation entre les pratiques entrepreneuriales, et celui-ci renvoie partiellement à la dimension dialectale. Pour exemple, l'enquête a souligné des pratiques commerçantes nettement structu- 
rées autour des réseaux locaux au sein des commerçants Chaozhous à Paris ${ }^{5}$ alors qu'elles sont plus nuancées pour ceux d'origine cantonaise. L'enquête révèle que si la grande majorité des entrepreneurs cantonais de Montréal $^{6}$ se passent de liens économiques transnationaux (10/12), ce n'est le cas que chez un peu moins de la moitié des vingt-cinq commerçants montréalais. Ils développent, en outre, un nombre plus important de liens locaux avec des entreprises de co-ethniques, soulignant de fait une pratique spécifique aux Cantonais de Montréal, par ailleurs souvent localisés dans le Chinatown, probablement en raison de l'ancienneté de cette communauté.

C'est dans le XIII ${ }^{\text {e }}$ arrondissement de Paris et dans le Chinatown de Montréal que nous avons rencontré le plus de commerces de proximité répondant spécifiquement à une demande " ethnique ". Les attentes concernent d'abord, la nourriture, avec de nombreux commerces alimentaires et quelques restaurants servant "ce que les Asiatiques mangent chez eux " (Mme Chen, employée de restaurant, Paris), viennent ensuite les médecins chinois et les herboristeries, la presse chinoise ou encore les agences de voyages spécialisées dans les pays d'Asie. Ce relevé correspond au niveau local décrit par Kloosterman et Rath (2001 : 197-198) : les voisinages à haute concentration de population immigrée sont des terrains fertiles pour ce type de commerce impliquant proximité et réseaux sociaux. Au facteur géographique s'ajoute le facteur numérique, l'importance des communautés chinoises de Paris et Montréal a permis la prolifération de ces commerces, alors que ceux-ci ne sont apparus que plus récemment à Bruxelles et ils y sont encore rares.

Enfin, dans les trois villes, la population se renouvelle. Ceci se remarque principalement à Paris et Montréal où de nouveaux flux migratoires se développent. Néanmoins, la population se dirigeant vers le Canada diffère de celle qui afflue vers la France. En effet, si le Canada est un pays d'immigration, sélectionnant ses nouveaux résidents en fonction de leur niveau de qualification (diplômes et expériences professionnelles), la capitale française semble plus fortement inscrite dans des réseaux de migrations irrégulières. Le phénomène des populations clandestines touchant la France et Paris a été décrit par Yun et Poisson (2005) et leurs travaux ont pu souligner que les ateliers clandestins y foisonnent et alimentent des commerces réguliers (magasins de vêtements, commerces alimentaires, restaurants). La population chinoise de Paris et Bruxelles est, par conséquent, moins éduquée et plus précaire que celle de Montréal. En outre, au Canada, plus nombreux sont les nouveaux venus qui, bénéficiant d'un capital de départ, investissent directement dans un commerce (Nicholls, 2011). L'évolution dans les trois villes s'explique également par le renouvellement naturel de la population, dans le cadre où des immigrés de deuxième génération reprennent parfois les commerces. Dans le " triangle de Choisy ", par exemple, le changement de population sera surtout visible d'ici une à deux décennies, suite au vieillissement de la population réfugiée et de la non-reprise des commerces par les enfants. Cependant, il n'est pas dit que la "Petite Asie " (Raulin, 2000) disparaisse ; des Chinois continentaux issus de vagues migratoires récentes, pour certains employés dans ces commerces, sont prêts à reprendre le flambeau.

5 Dix des quatorze Chaozhous sont parisiens, pour la plupart du XIlle arrondissement.

6 Douze des vingt Cantonais sont montréalais, cinq sont bruxellois et trois parisiens. 
La "Petite Asie " prendrait dès lors un caractère chinois plus marqué. Une évolution similaire pourra vraisemblablement s'observer dans le quartier chinois de Montréal où les Cantonais, descendants d'anciens immigrés et réfugiés, cèderont peu à peu la place aux nouveaux venus, majoritairement issus de Chine continentale.

\section{Évolution des commerces selon le secteur et le type de commerce}

Les pratiques économiques et leur évolution sont fortement liées au type de commerce et aux produits vendus. Les résultats d'enquêtes soulignent que le groupe de commerces chinois ne reposant sur aucun réseau ethnique est presque exclusivement composé de commerces de type " non-exotique " ou " occidental " (onze cas sur douze), la seule exception étant un take-away de nourriture chinoise installé sous forme d'une franchise et lié à une grande compagnie canadienne proposant différents types de nourriture exotique. Mais tous les commerces de type occidental ne se situent pas hors réseau ethnique: les grossistes wenzhous en maroquinerie et bijoux fantaisie importent leurs produits à prix avantageux auprès d'entreprises situées en Chine. II en va de même pour les magasins de vêtements. À ces dynamiques qui éclairent la diversité des structures économiques vient s'ajouter celle des commerces qui, par leur nature, ne dépendent pas de réseaux économiques ethniques, que ce soit au niveau national ou transnational. Il s'agit de la catégorie des activités commerciales que Waldinger nomme les "secteurs repris par les minorités". Ainsi, de nombreuses blanchisseries tenues par des Chinois se sont autrefois développées à Montréal et dans d'autres villes d'Amérique du Nord ; de même, dès la fin de la Seconde Guerre mondiale, les Wenzhous du IIIe arrondissement de Paris reprennent aux Juifs le secteur de la fabrication et de la vente en gros de maroquinerie et bijoux fantaisie (Live, 1992). Plus récemment, un nombre croissant de petites épiceries de Montréal sont rachetées par de nouveaux venus en provenance de la Chine continentale. L'investissement en masse dans ces secteurs a donc pour particularité de dépendre fortement de contingences historiques et du contexte économique, bien plus que le secteur ethnique qui, en dépit de ses évolutions, reste en lui-même une valeur relativement sûre. Les commerces de produits exotiques, quant à eux, entretiennent des liens économiques ethniques, majoritairement transnationaux (neuf sur les treize commerces concernés). C'est le cas des commerces alimentaires qui importent ou s'approvisionnent auprès d'ateliers de confection d'aliments tenus par des immigrés chinois. Nos répondants de Montréal, détaillants en thés et herbes, notamment, ne se satisfont pas de la seule importation des ateliers de fabrication de Vancouver, leurs produits nécessitent une importation internationale. Les magasins d'objets décoratifs chinois pratiquent également et presque toujours l'importation en provenance d'Asie et de Chine, excepté quelques commerces montréalais qui se fournissent en partie auprès d'ateliers de fabrication chinois installés à Toronto ou Vancouver.

Enfin, indépendamment du type de produits, certains commerces doivent leur succès à leurs fournisseurs en Chine. En effet, de plus en plus de commerces importent directement, sans passer par le grossiste et se voient avantagés par rapport à la concurrence qui se fournit selon l'ancien système. En raison de l'ouverture de la Chine et de la facilitation des importations, l'enquête 
laisse entrevoir de nouveaux comportements où des répondants indiquent avoir eu l'opportunité de créer une entreprise d'import-export en plus de leur activité de commerce de détail ou de restauration. Ceci n'est pas sans conséquence sur le secteur de la vente en gros. L'analyse des réponses obtenues auprès de grossistes et détaillants des trois villes fait apparaître un changement majeur qui s'opère depuis quelques années. Quelques grossistes se sont transformés en commerce de gros et détail afin de miser sur les deux tableaux. Mme Xu (Montréal), fait état de la situation actuelle :

"De plus en plus, comme la [conjoncture] de l'économie est très faible, et les concurrents [sont] très forts, la plupart des clients importent direct, pas besoin [d']intermédiaires. [...] On est [...] en train de [disparaître] dans la vente en gros [...]. Puis j'ai eu cette location, alors j'ai sauté dessus pour faire les deux. Un seul détail, ça ne marche pas, et un seul vente en gros, ça ne marche pas non plus ; les deux, c'est complémentaire.

[Maintenant] j'ai [aussi] les revenus des vêtements. Pourquoi j'ai fait des vêtements? [P] arce qu'on a plusieurs branches avant; les bibelots, ça ne marche pas du tout maintenant, à cause des grandes surfaces. Les gens [cherchent] des choses plus utiles, abordables... Alors dans mon cas, j'ai une boutique, mais très petite parce que j'ai besoin de l'entrepôt et j'ai pensé que ça, c'est vendable, et en même temps, ça ne prend pas beaucoup de place pour entreposer, et pour le shipping, le transport international [...]. "

Un peu plus loin dans l'entretien, elle dit envisager un autre système qui abandonnerait la vente en gros:

"[J]e veux ouvrir trois magasins. Je n'ai pas besoin de vendre en gros, je veux faire une sorte de franchise. Pour importer direct et vendre dans mes trois magasins.

Mais, ça s'annonce difficile, je pense.

Oui, la concurrence est très forte. "

Son témoignage explique aussi pourquoi les liens économiques entre ces entreprises et certains pays d'Asie s'affaiblissent au bénéfice de la Chine où les produits sont meilleur marché. Ainsi, elle affirme s'être approvisionnée, entre 1992 et 2002, dans différents pays d'Asie, dont les Philippines, la Thaïlande et Hong Kong, avant de s'approvisionner à $100 \%$ en Chine, ceci en raison du moindre coût de la marchandise. Ce témoignage est remarquable au niveau des stratégies d'adaptation énumérées : le choix des pays d'importation ; les pratiques de vente (vente qui se fait désormais au détail en plus de la vente en gros) ; la gamme de produits proposés (qui se diversifie) ; et enfin, le projet d'utiliser le système de franchise pour faire fructifier son commerce. Nous voyons, dans le discours de cette patronne, une volonté de se maintenir et de s'améliorer par ces diverses stratégies, preuve du caractère dynamique et malléable de la pratique du commerce.

Une évolution est aussi perceptible dans le discours des Wenzhous du $\mathrm{III}^{\mathrm{e}}$ arrondissement comme le montre le témoignage de la fille d'un couple de grossistes :

"[M]aintenant, ce n'est plus pareil qu'il y a vingt ans ou trente ans [...] : à l'époque le commerce était beaucoup plus facile, il y avait moins de concurrence, et puis, je veux dire, n'importe quoi se vendait, il n'y avait pas trop de difficultés... et puis là, on a besoin de choses très très ciblées. Et puis bon, la plupart des grandes entreprises se fournissent 
directement en Asie maintenant; ils n'ont plus besoin de faire appel [...] aux grossistes, quoi. " (Tang Yin)

L'adaptation peut également consister en l'ajout d'un commerce de détail à un commerce de gros:

"Avant 1990, nous faisions grossistes et la rue était plus petite.

Puis après, il y a eu des travaux pour agrandir la rue. Nous, on continuait à faire grossistes, mais ça ne marchait plus comme avant : ils changeaient les routes, et il n'y avait plus de commerce à faire. Nos chiffres d'affaires ont beaucoup baissé... c'était difficile! Des gens venaient, mais c'était pour du détail et on ne pouvait rien leur vendre. Et comme ça le chiffre d'affaires a continué à baisser, baisser, jusque la fin de l'année 2004 où nous avons changé le commerce [...]. Maintenant, on fait les deux : vente en gros et détail. Comme ça, ça marche ! Et ça va bien, ça reprend! " (Mme Zheng)

Autre tendance récente chez les Wenzhous du III ${ }^{\mathrm{e}}$ arrondissement : I'apparition dans certaines maroquineries d'objets pour touristes fabriqués en Chine qu'achètent des revendeurs de minorités non chinoises pour les écouler sur les lieux touristiques. Ce commerce s'ajoute à celui des produits traditionnellement vendus dans ce quartier (maroquinerie et bijoux de fantaisie).

\section{Conclusion}

Comme Granovetter (1985) ou, plus récemment, Kloosterman et Rath (2003 ; 2001), nous partons du principe que la sphère économique fait partie intégrante du social, et que toute action économique, comme la création d'un commerce et les stratégies développées en vue du fonctionnement de celui-ci, est encastrée dans une série de faits sociaux. En découle une imbrication de rapports d'ethnicité, de rapports familiaux, de rapports sociaux de sexe, et plus globalement de rapports de pouvoir et aux enjeux multiples. En d'autres termes, créer un commerce n'implique pas uniquement une quête de gain financier, mais aussi une recherche de reconnaissance sociale via l'acquisition d'un statut et d'une identité professionnelle, voire un besoin de sociabilité et de cohésion sociale, car l'entrepreneuriat peut aussi être une affaire de famille et de réseau ethnique (Nicholls, 2012).

L'entrepreneuriat ne se limite cependant pas à cela. Suite à notre enquête, nous souscrivons aux conclusions de Menkhoff et Sikorski (2002) quant à la tendance, chez les entrepreneurs, à la cosmopolitisation et à la rationalité instrumentale. En effet, le principal apport de cette recherche réside en la confirmation de l'importance des réseaux transnationaux et la part grandissante de la Chine continentale dans cet ensemble. Nos conclusions rejoignent celles de Bousetta et Martiniello (2008) sur l'accroissement des activités économiques transnationales depuis le début des années 1990, essentiellement en direction de la Chine, rendues possibles par son ouverture et par l'accélération de la mondialisation des échanges économiques. Cet aspect, allant de pair avec les nouveaux moyens de transport et de communication, générateurs d'échanges et de proximité entre les Chinois dispersés dans le monde, transforme progressivement les communautés locales, ses commerces et ses pratiques. Cette évolution se remarque également dans la différence de pratiques observée entre les entrepreneurs selon l'âge : les jeunes générations d'entrepreneurs établissant davantage de 
liens transnationaux. Depuis son ouverture, la Chine devient le pays d'importation de prédilection en raison du coût avantageux de ses produits. En cela, notre enquête vient affirmer l'idée le choix du pays d'importation n'est pas uniquement sous-tendu par l'aspect identitaire, mais qu'il est aussi, et surtout, guidé par un choix rationnel.

Comme nous l'avons souligné dans nos précédents travaux (Nicholls, 2012), la dimension communautaire n'est pas seulement un phénomène culturel et identitaire, mais elle est à la base d'une véritable stratégie fondée sur un capital social et culturel que l'acteur utilise et qui s'actualise via les réseaux sociaux de co-ethniques. Nous devons envisager le commerçant chinois comme un acteur social et son ethnicité selon une perspective dynamique, à savoir que cette appartenance est appropriée et utilisée de manière stratégique. Ces stratégies ne peuvent toutefois se concevoir que dans un contexte mondial reposant sur une main-d'oeuvre précaire et peu exigeante. Des produits bon marché venus de Chine et d'Asie ne sont possibles qu'en vertu d'une situation mondiale de répartition inégale des ressources entre les pays. Pour paraphraser Tarrius (2002:66), ces commerçants de la diaspora chinoise prospèrent en exploitant, d'une part, la situation de la Chine et des autres pays d'Asie, en particulier la pauvreté et les mauvaises conditions de travail qui y sont attachées, d'autre part, la richesse de leur société d'accueil et le budget loisirs de leurs nationaux. Néanmoins, il peut exister des avantages pour le pays d'origine : I'envoi d'une partie des salaires et le chiffre d'affaires des entreprises d'exportation en constituent des exemples. L'opportunité économique que représente l'ouverture de la Chine a un impact considérable sur la pratique du commerce des immigrés chinois. De plus en plus de magasins importent directement, ce qui nécessite une bonne connaissance des ateliers de fabrication et des entreprises d'exportation basés en Chine et implique la création de liens économiques entre la Chine et les divers pôles de la diaspora. Corollairement, les liens locaux s'affaiblissent comme en témoignent les difficultés rapportées par les grossistes de Paris et Montréal.

Les commerces évoluent différemment selon les villes par l'adaptation d'anciens secteurs et l'apparition de nouveaux types d'activités. Ces changements sont liés à une multiplicité de facteurs : le rapport au majoritaire dans le pays d'accueil et la perception que celui-ci a du groupe " chinois ", la situation économique et politique des pays d'origine, le développement des échanges transnationaux, des moyens de transport et de communication et, plus largement, I'apparition d'une culture urbaine mondiale facilitant l'insertion des nouveaux immigrés. Malgré des similitudes observées dans les trois villes qui pourraient, donner lieu à des interprétations culturalistes, I'adaptation au contexte est omniprésente. Les expressions de cette capacité sont nombreuses : qu'il s'agisse de changer le pays d'importation lorsque la situation de la Chine y est favorable et d'y établir des liens avec de nouveaux fournisseurs ou de diversifier ou de modifier ses produits selon la demande, de travailler avec des Chinois d'autres dialectes par nécessité, d'inclure des employés non-chinois dans l'entreprise et aussi de faire preuve de davantage d'autonomie dans un monde plus individualiste. Les transformations observées chez les nouvelles générations urbaines et éduquées illustrent parfaitement ce phénomène de réinterprétation continuelle de l'identité ethnique. En tous les cas, loin du stéréotype d'immuabilité qui lui est souvent attribué, le commerce chinois subit de nombreuses transformations, visibles ou invisibles, rapides ou progressives. 
Pour aller au-delà, des limites de cette étude, des recherches spécifiques sur les différents groupes dialectaux et les rapports que ceux-ci entretiennent entre eux seraient nécessaires. De plus, si la variable "genre " a été prise en considération, elle a donné peu de résultats ; des recherches plus poussées sur les différences de situations et de pratiques entre entrepreneurs hommes et femmes devraient être menées. Enfin, un dernier axe de recherche viserait l'approfondissement de la question des transformations observées chez les nouveaux venus d'une Chine en pleine mutation, qui promettent de changer sensiblement les pratiques et l'image du commerçant chinois de la diaspora.

\section{Références bibliographiques}

Auster Ellen and Aldrich Howard (1984) Small Business Vulnerability, Ethnic Enclaves and Ethnic Entreprise, in Robin Ward and Richard Jenkins Eds., Ethnic Communities in Business, Cambridge, Cambridge University Press, pp. 39-54.

Barth Fredrik (1995) [1969] Les groupes ethniques et leurs frontières, in Philippe Poutignat et Jocelyne Streiff-Fenart, Théories de l'ethnicité, Paris, PUF, pp. 203-249.

Bastenier Albert (1992) L'État belge face à l'immigration : les politiques sociales jusqu'en 1980, Louvain-La-Neuve, Academia, 166 p.

Bastenier Albert (2004) Qu'est-ce qu'une société ethnique ? Ethnicité et racisme dans les sociétés européennes d'immigration, Paris, PUF, 348 p.

Berbagui Dalila (2005) Commerce et petite entreprise étrangère dans la ville (1980-2002), Ethnologie française, 35 (1), pp. 109-115.

Berger Brigitte (1991)The Culture of Modern Entrepreneurship, in Brigitte Berger Éd., The Culture Entrepreneurship, San Francisco, ICS Press, pp. 13-32.

Bonacich Edna (1973) A Theory of Middleman Minorities, American Sociological Review, 38, pp. 583-594.

Bourdieu Pierre (2006) [1980] Le capital social. Notes provisoires, in Antoine Bevort et Michel Lallement Éds., Le capital social. Performance, équité et réciprocité, Paris, La Découverte, pp. 31-34.

Bousetta Hassan et Martiniello Marco (2008) Les pratiques transnationales des immigrés chinois et marocains de Belgique, Revue européenne des migrations internationales, 24 (2), pp. 45-66.

Cardinal Linda et Couture Claude (1998) L'immigration et le multiculturalisme au Canada: la genèse d'une problématique, in Manon Tremblay Éd., Les politiques publiques canadiennes, Québec, Presses de l'université Laval, pp. 239-264.

CECLR (Centre pour l'égalité des chances et la lutte contre le racisme) (2005) L'immigration chinoise en Belgique. Principales évolutions et perspectives, [en ligne] consulté le 10/06/2012. URL : http://www.diversite.be/?action=publicatie_ detail\&id=36\&thema $=4$

Cha Jonathan (2004) La représentation symbolique dans le contexte de la mondialisation. L'exemple de la construction identitaire du quartier chinois de Montréal, JSSAC / JSÉAC, 29 (3), pp. 3-18. 
Coleman James (1988) Social Capital in the Creation of Human Capital, American Journal of Sociology, 94, pp. S95-S120.

Dyer Linda and Ross Christopher (2000) Ethnic Enterprises and Their Clientele, Journal of Small Business Management, 38 (2), pp. 48-66.

Godin Jean-François (2005) Immigrants et travail à Montréal : la dynamique de l'établissement professionnel des dix premières années, thèse de doctorat en sciences humaines appliquées, Jean Renaud dir., Montréal, université de Montréal, non publié, $236 \mathrm{p}$.

Granovetter Mark (1985) Economic Action and Social Structure: The Problem of Embededness, American journal of sociology, 91 (3), pp. 481-510.

Granovetter Mark (2006) L'influence de la structure sociale sur les activités économiques, Sociologies pratiques, 13 (2), pp. 9-36.

Greenhalgh Susan (1994) De-Orientalizing the Chinese Family Firm, American Ethnologist, 21 (4), pp. 746-773.

Hassoun Jean-Pierre (1993) Des patrons 'chinois' à Paris. Ressources linguistiques, sociales et symboliques, Revue française de sociologie, 34 (1), pp. 97-123.

Hullebroek Patrick (1992) La politique générale d'immigration et la législation sur les étrangers, in Anne Morelli Éd., Histoire des étrangers et de l'immigration en Belgique de la préhistoire à nos jours, Bruxelles, Vie Ouvrière, pp. 119-140.

Juteau Danielle (1983) La production de l'ethnicité ou la part réelle de l'idéel, Sociologie et sociétés, 15 (2), pp. 39-54.

Juteau Danielle (1996) L'ethnicité comme rapport social, Mots. Les langages du politique, 49, pp. 97-105.

Juteau Danielle, Daviau-Guay Jocelyne et Moallem Minoo (1999) L'entrepreneurship ethnique à Montréal : première esquisse, Cahiers Québécois de démographie, 21 (2), pp. 119-145.

Kaufmann Jean-Claude (1996) L'entretien compréhensif, Paris, Nathan, 128 p.

Kloosterman Robert and Rath Jan (2001) Immigrant Entrepreneurs in Advanced Economies: Mixed Embeddedness Further Explored, Journal of Ethnic and Migration Studies, 27 (2), pp. 189-201.

Kloosterman Robert and Rath Jan (2003) Introduction, in Robert Kloosterman and Jan Rath Eds., Immigrant Entrepreneurs: Venturing Abroad in the Age of Globalization, Oxford, Berg, pp. 1-16.

Kymlicka Will (1995) Multicultural Citizenship: A Liberal Theory of Minority Rights, Oxford, Oxford University Press, 288 p.

Li Peter (1988) The Chinese in Canada, Toronto, Oxford University Press, 192 p.

Li Peter (1993) Chinese Investment and Business in Canada: Ethnic Entrepreneurship Reconsidered, Pacific Affairs, 66 (2), pp. 219-243.

Light Ivan (1972) Ethnic Enterprise in America. Business and Welfare Among Chinese, Japanese, and Blacks, Berkeley, Los Angeles, London, University of California Press, 210 p. 
Light Ivan and Karageorgis Stavros (1994) The Ethnic Economy, in Neil Smelser and Richard Swedberg Eds., The Handbook of Economic Sociology, Princeton, Princeton University Press, pp. 647-671.

Live Yu-Sion (1992) Les Chinois de Paris depuis le début du siècle. Présence urbaine et activités économiques, Revue européenne des migrations internationales, 8 (3), pp. 154-174.

Madoui Mohamed (2006) Entrepreneurs d'origine maghrébine en France et capital social, in Antoine Bevort et Michel Lallement Eds., Le capital social. Performance, équité et réciprocité, Paris, La Découverte, pp. 246-262.

Ma Mung Emmanuel (2000) La diaspora chinoise, géographie d'une migration, Paris, Ophrys, $176 \mathrm{p}$.

Ma Mung Emmanuel (2009) Le prolétaire, le commerçant et la diaspora, Revue européenne des migrations internationales, 25 (1), pp. 97-118.

Martiniello Marco (1993) Pour une sociologie politique de la, situation postmigratoire en Belgique, in Marco Martiniello et Marc Poncelet Éds., Migrations et minorités ethniques dans l'espace européen, Bruxelles, De Boeck université, pp. 167-185.

Menkhoff Thomas and Sikorski Douglass (2002) Asia's Chinese Entrepreneurs Between Myth-Making and Renewal, in Thomas Menkhoff and Solvay Gerke Eds., Chinese Entrepreneuship and Asian Business Networks, New-York, RoutledgeCurzon, pp. 23-42.

Milza Pierre (1985) Un siècle d'immigration étrangère en France, Vingtième Siècle. Revue d'histoire, 7, pp. 3-17.

Morokvasic Mirjana (1991) Roads to Independence. Self-Employed Immigrants and Minority Women in Five European States, International Migration, 29 (3), pp. 407-420.

Nicholls Alexander (2011) Pratique du commerce et rapports d'ethnicité en situation d'immigration. Le cas des Chinois de Paris, Bruxelles et Montréal, thèse de doctorat en Sciences politiques et sociales, Louvain-la-Neuve, université catholique de Louvain, non publié, $584 \mathrm{p}$.

Nicholls Alexander (2012) Capital social ethnique et entrepreneuriat. Le cas des commerçants chinois de Paris, Bruxelles et Montréal, Sociologie, 3 (4), pp. 395-411.

Noiriel Gérard (2001) État, nation et immigration. Vers une histoire du pouvoir, Paris, Belin, 399p.

Nonini Donald and Ong Aiwha (1997), ChineseTransnationalism as an Alternative Modernity, in Donald Nonini Donald and Aiwha Ong Eds., Ungrounded Empires. TheCultural Politics of Modern Chinese Transnationalism, New York \& London, Routledge, pp. 3-36.

Pang Ching Lin (2002) Business Opportunity or Food Pornography? Chinese Restaurant Ventures in Antwerp, International Journal of Entrepreneurial Behaviour \& Research, 8 (1), pp. 148-161.

Pécoud Antoine (2004) Réseaux, ethnicité et institutions dans les économies immigrées, Hommes \& Migrations, 1250, pp. 13-23.

Pécoud Antoine (2012) Immigration, entrepreneuriat et ethnicité», Métropoles, 11, 20 p., [En ligne] consulté le 01/08/2013. URL : http://metropoles.revues.org/4560 
Phizacklea Annie (1988) Entrepreneurship, Ethnicity, and Gender, in Sallie Westwood and Parminder Bhachu Eds., Enterprising Women. Ethnicity, Economy, and Gender Relations, London, Routledge, pp. 20-33.

Portes Alejandro (1998) Social Capital: Its Origins and Applications in Modern Sociology, Annual Review of Sociology, 24 (1), pp. 1-24.

Poutignat Philippe et Streiff-Fenart Jocelyne (1995) Théories de l'ethnicité, Paris, PUF, $270 \mathrm{p}$.

Raulin Anne (2000) L'ethnique est quotidien. Diasporas, marchés et cultures métropolitaines, Paris, L'Harmattan, 229 p.

Redding Gordon (1991) Culture and Entrepreneurial Behavior among the Overseas Chinese, in Brigitte Berger Ed., The Culture Entrepreneurship, San Francisco, ICS Press, pp. 137-156.

Salaff Janet et al. (2003) Ethnic Entrepreneurship, Social Networks, and the Enclave, in Brenda Yeoh, Tong Chee Kiong and Michael Charney Eds., Approaching Transnationalism : Transnational Societies, Multicultural Contacts, and Imaginings of Home, Boston, Kluwer Academic Publishers, pp. 61-82.

Schnapper Dominique (1994) La communauté des citoyens. Sur l'idée moderne de nation, Paris, Gallimard, 230 p.

Smart Joséphine (2003) Ethnic Entrepreneurship, Transmigration, and Social Integration: An Ethnographic Study of Chinese Restaurant Owners in Western Canada, Urban Anthropology and Studies of Cultural Systems and World Economic Development, 32 (3), pp. 311-342.

Statistique Canada (2006) Ethnocultural Portrait of Canada Highlight Tables, 2006 Census, [en ligne] consulté le 25/05/2011. URL : http://www12.statcan.ca/ census-recensement/2006/dp-pd/hlt/97-562/index.cfm?Lang=E

Tarrius Alain (2002) La mondialisation par le bas. Les nouveaux nomades de l'économie souterraine, Paris, Balland, 178 p.

Taylor Charles (1994) Multiculturalisme, différence et démocratie, Paris, Flammarion, 144p.

Volery Thierry (2007) Ethnic Entrepreneurship: A Theoretical Framework, in Leo-Paul Dana Ed., Handbook of Research on Ethnic Minority Entrepreneurship, Cheltenham, Edward Elgar, pp. 30-41.

Waldinger Roger, Aldrich Howard and Ward Robin (1990) Opportunities, group characteristics and strategies, in Roger Waldinger, Howard Aldrich and Robin Ward Eds., Ethnic Entrepreneurs: Immigrant Business in Industrial Societies, London, Sage, pp. 13-48.

Wilson Kenneth and Portes Alejandro (1980) Immigrant Enclaves: An Analysis of the Labor Market Experiences of Cubans in Miami, American Journal of Sociology, 86, pp. 295-319.

Wirth Louis (1945) The Problems of Minority Groups, in Robert Linton Ed., The Science of Man in the World Crisis, New York, Columbia University Press, pp. 347-372. 
Yun Gao et Poisson Véronique (2005) Le trafic et l'exploitation des immigrants chinois en France, Genève, Bureau International du Travail, 142 p.

Zalc Claire (2001) Petits entrepreneurs étrangers en ville. Localisations urbaines, réseaux migratoires et solidarités professionnelles dans la Seine pendant I'entre-deux-guerres, Histoire urbaine, 2 (4), pp. 67-82.

Zalc Claire (2010) Melting Shops. Une histoire des commerçants étrangers en France, Paris, Perrin, $330 \mathrm{p}$. 


\section{Nicholls Alexander}

\section{Les pratiques économiques des entrepreneurs issus de l'immigration chinoise. Le cas de Paris, Bruxelles et Montréal}

Cet article explore les liens économiques établis par les entrepreneurs issus de l'immigration chinoise. II se base sur une analyse qualitative de soixante entretiens. Les personnes interrogées sont des Chinois en provenance de divers pays (République populaire de Chine, Taïwan, Vietnam, Laos, Cambodge, Thaïlande, Indonésie, Madagascar), établis dans les villes de Paris, Bruxelles et Montréal. Les données empiriques ici présentées indiquent que le phénomène est complexe et contextuel, et que les pratiques en matière d'échanges économiques entre les entreprises sont variées. L'article en étudie l'aspect ethnique, selon les caractéristiques du patron et la ville d'implantation, ainsi que leur évolution dans le temps. La recherche conclut à l'importance du facteur générationnel dans la pratique du commerce et à la part croissante des échanges transnationaux, en particulier en direction de la Chine. Corollairement, les échanges locaux entre entreprises chinoises immigrées s'affaiblissent. Loin du stéréotype d'immuabilité qui leur est parfois attribué, les entrepreneurs chinois immigrés font preuve d'adaptation aux contextes local, national et transnational.

\section{Economic practices of Chinese immigrants entrepreneurs. The case of Paris, Brussels and Montreal}

This article explores the economic ties established by entrepreneurs from ethnic Chinese immigration, based on a qualitative analysis of sixty interviews. The interviewees are Chinese from various countries (People's Republic of China, Taiwan, Vietnam, Laos, Cambodia, Thailand, Indonesia, Madagascar), established in the cities of Paris, Brussels and Montreal. The empirical data presented here indicate that the phenomenon is complex and context-dependant, and that the practices of economic exchanges between businesses are diverse. The paper examines their ethnic aspect, depending on the characteristics of the business owner and the city, and their evolution over time. The research stresses the importance of generational factor in business practices and the increasing role of international trade, particularly towards China. As a corollary, local exchanges between immigrant Chinese companies are weakening. Far from the stereotype of immutability that has sometimes been attributed to them, the Chinese immigrant entrepreneurs demonstrate adaptation to local, national and transnational contexts.

\section{Las prácticas económicas de los empresarios de la inmigración china. El caso de París, Bruselas y Montreal}

El presente artículo explora los vínculos económicos establecidos por empresarios de la inmigración de origen chino, basándose en el análisis de 60 entrevistas. Las personas entrevistadas son nacionales chinos en procedencia de diversos países (República Popular de China, Taiwán, Vietnam, Laos, Camboya, Tailandia, Indonesia y Madagascar) y establecidos en las ciudades de París, Bruselas y Montreal. Los datos empíricos que se presentan indican que el fenómeno es complejo y contextual, y que las prácticas en materia de intercambios económicos entre las empresas son diversas. El artículo estudia el aspecto étnico, atendiendo a las características del propietario y de la ciudad de implantación, 
así como su evolución temporal. La investigación establece la importancia del factor generacional en la práctica del comercio y la creciente implantación de intercambios transnacionales, en particular en dirección de China. Por el contrario, los intercambios locales entre empresas inmigrantes chinas se están debilitando. Lejos del estereotipo de inmutabilidad que se les atribuye, los empresarios inmigrantes chinos demuestran su capacidad de adaptación a los contextos local, nacional y transnacional. 